warehouses and manufactories-to the merchant, whose dealings are in filamentous substances, such as wool, flax, \&c. the instrument may render great assistance; the weight of all such substances varying considerably with their exposure to an atmosphere more or less dry; also in malting-houses, and in the laboratory of the chemist. The same might be said in regulating the temperature and humidity of public hospitals. ${ }^{*}$ At a few of these valuable institutions, hygrometrical observations are carefully recorded, but still not upon that extensive scale that the importance of the subject demands. Every dormitory attached to our hospitals, prisons, workhouses, schools, \&c., in fact, wherever a number of persons congregate, whether these apartments are used for sleeping or working in, hygrometrical observations should be taken, in order that these places may be kept in the most favourable condition for sustaining life. We have not the least doubt that if these suggestions are acted upon, a very material decrease in some diseases (pulmonary especialiy) will result therefrom.

Glaisher"s Stand to Car"y the Thermometers. It is desirable that the thermometers should be suspended so that their bulbs be freely exposed to the air on all sides, at the distance of four feet above the soil, and removed to some distance from houses or water, or from any object which might reflect heat to them. To attain these conditions it is necessary to have a stand to carry the instruments. Annexed is the form of a suitable re volving stand; the instruments are to be placed on its face, and the back turned towards the sun.

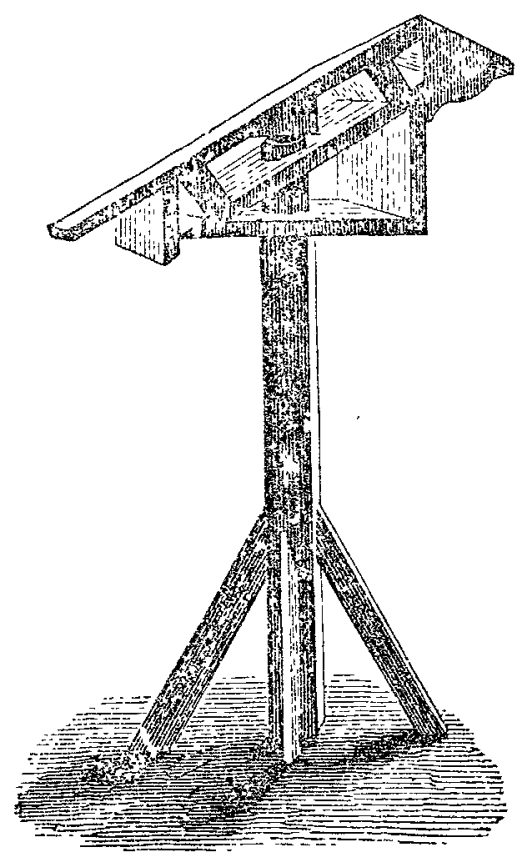

Glaisher's stand to carry the thermometers.

Ozone Test Paper. - The peculiar gas ozone is generated from the oxygen of the air when it is surcharged with electricity. Ozone possesses a powerful odour, while ordinary oxygen gas does not: the former, when in excess, is a violent poison; the latter an indispensable supporter of animal life. The presence of ozone in excess exerts a very sensible influence in many ways upon health, and in its chemical activity in changing the character of various substances used in domestic life. The inhalation of an irritating gas cannot but prodnce injurious effects on organs so delicate as the lungs; and perhaps many of the now anomalous and inexplicable effects of change of air to patients suffering from chest diseases may hereafter receive their solution in a more intimate acquaintance with the laws of ozone. The eminent chemist Schonbein invented a delicate test paper, $*$ imbued with a mixture of iodide of potash and starch, by means of which the medical man can detect the presence of this gas, and estimate the quantity contained in the air.

Anemometer-Direction and Strength of the Wind.-The force and direction of the wind claim the attention of the me-

* See Glaisher's valuable "Hygrometrical Tables," published by Taylor and Francis, Red Lion-court, Fleet-street, London.

* These test papers are made as follows:-To one part of iodide of potassium and ten parts of starch add 200 parts of distilled water; boil together for a few seconds, then dip slips of white bibulous paper into the solution; when dry they are ready for use. If the paper is hung in any situation where a free current of air cireulates, but not sunshine, the colour will be found to change to a pale blue tinge, as the ozone is more or less powerful; and, on the con trary, if none be present, it remains colourless. teorologist. For this purpose several anemometers have been constructed that have been found to answer well, and to act a considerable time without derangement. It is, however, an expensive instrument, and difficult to adjust in a suitable position. There are few observers in possession of a locality for the placing an anemometer to give a really true indication of the movements of the air. It is, however, an instrument applying to a meteorological observatory, and as such we may mention Osler's anemometer, which itself registers the direction of the wind, its pressure in pounds on the square foot, and the fall of rain. It is furnished with a clock which drives a traversing-board, upon which is fastened a paper ruled with hour lines, upon which pencils, in comnexion with the vane, pressure-plate, and rain-receiver, register these elements as the clock drives the traversing board from one hour-line to the next.

In the absence of an anemometer, the direction of the wind may be referred to the position of a vane, or, if no good vane is in sight, the course of the clouds or smoke show the direction, if the position (to the observer) of the points of the compass be known.

The Evaporator. - This consists of a short cylinder, twelve inches in diameter, having connected with it, by means of a stopcock, a glass tube, graduated to hundredths, and terminating in a lower vessel, which will contain a sufficient quantity of water to be raised by artificial pressure into the upper one for exposure to the atmosphere.

The Electrometer. - The simplest is Glaisher's, which, being portable, should become generally adopted. Atmospheric electricity has been much neglected by metuorologists; nevertheless it is an important item of meteorological investigation. It is recommended by Mr. Glaisher that the continuity of the observations be preserved; the positions of the different instruments should not be changed, nor the method of reading and registering altered; that punctuality be observed for the observations, and the instruments be read off the same way daily. The thermome. ters should be protected from rain; and in making a reading, the observer should do it quickly, and, whilst so doing, avoid touching or breathing on, or in any way warming, the thermometer by approaching too near. The direction and force of a gale of wind should be noted; the height of the barometer during its continuance; also, in thunder storms, the direction in which they move, and of the wind before, after, and during its continuance; the time when the electrical breeze springs up; the commencement and termination of rain; whether hail falls; and any other feature that may appear remarkable, or be deemed desirable to record. The times of breaking up of long dry periods and frosts, the termination of rainy periods, the commencement and duration of fogs, wind changes, \&c. In this way most valuable results will be accumulated.

\section{SUCCESSFUL CASE OF}

LIGATURE OF THE EXTERNAL ILIAC ARTERY, CLOSE TO ITS ORIGIN FROM THE COMMON ILIAC, FOR INGUINAL ANEURISM.

SEPARATION OF THE LIGATURE OY THX FORTY-SIXTH DAY.

Bx WM. PHILPOT BROOKES, M.D., F.R.C.S., SURGIION TO THE GBNRRAY HOSPITAL AND DISPENSARX, CHELTEMHAM.

JOSEPA S-, aged fifty-seven, cabinet-maker, residing at Cheltenham, of a spare cachectic habit, looking some years older than he is; of a gouty tendency; arcus senilis very distinctly marked; the heart and other visceral organs quite healthy, states that two years since, while using his brace to drill a bedstead, the head of which pressed upon the right groin in working it, on a sudden he felt great pain in the groin, and for a month afterwards he could seldom walk. A swelling appeared in the part; this for a time seemed improving, and caused him so little pain, that he consulted no surgeon till some six months after the accident. He again found much pain, and, upon closely examining the groin, discovered a small swelling, which he could hardly touch from the acuteness of the sensation. It now pulsated strongly; this gradually increasing in size, he became alarmed, and consulted 
Mr. Charles Gregory, surgeon, who at once discovered the character of the tumour, and desired me to see him.

Feb. 6th, 1856.- -Upon examination of the patient, I found an aneurism in the right inguinal region, the size of a turkey's egg, pulsating very forcibly; its coverings were tense, but sound, not discoloured. It had considerably forced Poupart's ligament upward out of its natural position. Pressure on the external iliac artery gave so much acute pain, that he could not allow it, the pressure but slightly restraining the pulsation in the aneurism. There was no aneurismal indication in any other part of the body, the general health being good, although he looked some years older than he was. On a careful consideration of all the points of the case, I felt satisfied that treatment by pressure could not be carried out. I determined to tie the external iliac artery as near its origin to the common iliac as could with safety be accomplished to ensure a sufficient clot.

The patient readily gave his consent, and on the 13 th of February, 1856, in the presence of numerous professional friends, I performed the operation at his residence. Making a semilunar incision, of five inches in length, commencing somewhat above the anterior-superior spinous process of the ilium, and extending to within an inch of the pubis, its convexity looking downwards, I dissected this flap; then dividing the aponeurosis of the external oblique muscle, reflecting it upwards, and passing my finger through the fasciæ transversalis, coming upon the sac of the peritoneum, I carefully pushed it upwards and inwards as far as possible, and reached the artery. I was now compelled to use the retractors, to keep the parts clear. The division of a small arterial branch, which bled most profusely, gave some trouble; one end being ligatured and still continuing to bleed from the other, an assistant kept pressure upon it. I secured the external iliac, after carefully separating it from its accompanying vein with my fingernail. Tied the vessel as high up, and as far from the diseased portion of the artery, as I possibly could. My ligature was waxed, thick silk. Tying the artery with a double knot, all pulsation in the tumour immediately ceased. Both ends of the silk were brought out, and the wound united with sutures. During the administration of chloroform, which was kindly given by Mr. Hartley, the resident surgeon of our hospital, he became much excited; had, however, no knowledge of the operation until placed in bed, and some minutes had elapsed after its completion; his delight was not small when told all was over. The limb was enveloped in flannel, a warm brick kept to his foot, bags filled with baked bran were placed down each side of the thigh and leg, and the extremity was kept bent upon the pelvis. - Nine P.M.: Pulse 92, and quiet; tongue moist; foot and leg warm; feeling in it as acute as ever; complains of slight tlatulence; no abdominal tenderness. He begged to be allowed a pipe, having for years been accustomed to smoke several during the day, and saying, "I shall not sleep unless I have one." I consented, and he rorked away at it most heartily. He also took a cup of gruel. The aneurismal tumour in the groin is not perceptible; no kind of pulsation can be felt by most careful examination.

14th. - Pulse 72, and good; tongue moist; slept but little; no abdominal pain. The foot and leg being warmer than the other, I merely kept the warm brick to the foot, and left off the flannel and bran bags. A slight pulsation has returned in the groin.

15th.-Slept well all night; pulse 82, soft; tongue clean; the bowels not acted npon since the operation; the wound united by the first intention. All other symptoms favourable. The aneurismal tumour is somewhat returned, and pulsates slightly. No trace of pulsation in the femoral or popliteal could be discovered.

17th. - The patient is progressing favourably; foot and leg keep warm; the sutures taken out of the wound, which is perfectly united; the tumour larger, pulsating more; pressure over the situation of the femoral, below the tumour, entirely controls the pulsation in the aneurism. There was some discoloration and œdema over the posterior and upper part of the ilium to-day; warm fomentations readily dispelled it. The bowels freely opened. Has been kept on low diet until this day, when some meat was allowed him.

19th. - He continues to go on well. The tumour still pulsates, and appears to receive its supply of blood by a retrograde circulation, as pressure on the femoral (which can now be discovered pulsating feebly just below the aneurism, but in no other portion of its course) entirely controls the pulsation through the tumour. No throb can be felt in the artery above the aneurism, or in the popliteal space. He complains to-day of some pain in the leg and knee; this he attributes to the severe frosty weather, which set in last night. He states that the cold tries him much; and we cannot keep the temperature of his room high enough for him. To take twenty drops of tincture of opium. This procured him a good night's rest, and all pain in the left knee disappeared.

23rd.-Going on well. It was found necessary to reduce his diet, as the meat disagreed, and he now takes it every other day. The aneurism still pulsates, but is getting smaller. Upon coughing, states that he felt a darting shooting pain in the situation of the ligature.

29th.-Going on very well. Aneurismal tumour pulsates; it is becoming much harder and smaller. He can now, for the first time for some months, lie over upon his left side without pain; this he could not attempt prior to the artery being ligatured, and the slightest pressure on the aneurism gave him intense agony; but since the operation that has entirely ceased. To have the whole of the leg and thigh bandaged, using slight pressure over the groin.

March 5th. - Gently tried the ligature without any result. The aneurism is harder, much diminished in size, and pulsates less. The bandaging to be kept up.

9th. - The pulsation in the aneurism has been gradually getting weaker; the sac more consolidated, and yesterday, in a moment, at two o'clock, all pulsation suddenly ceased. The man says it was so sudden that it startled him, and he pulled his watch from under his pillow to observe the time. I fancy the ligature gave slightly to-day. He complains that during the night his foot felt numbed. It was wrapped in flannel, and the warm brick applied to it; in fact, the brick has been rarely discontinued since the operation. I also had some baked bran applied to the leg. The bowels are costive, and require castor oil occasionally; the diet to be improved; to take mest twice a day.

12th. - No pulsation returned; the tumour gradually becoming absorbed; foot and leg quite comfortable; appetite good. Bandaging of the limb discontinued since the 9 th.

15th. - Yesterday he found a pain in the knee, which he persists is rheumatism; there was also a slight reddish blush around the situation of the aneurism. To-day, upon my visit, I found erysipelas and odema around the upper part of the thigh and groin, involving the aneurism, which is most painful upon the slightest touch. I requested him to carefully avoid handling the part, or permitting any of the medical gentlemen, who take an interest in the case, and visit him, to touch it. The tongue was clean and moist; pulse calm; bowels freely opened; little or no constitutional irritation. The thigh was dressed with lint dipped in a warm solution of poppyhead infusion, and the wet lint was covered with oil silk. For the first time since the operation, I allowed him some beer. The erysipelas did not extend around the ligature or cicatrix of the wound.

16th. - Countenance anxious; œdema and erysipelas much subsided; tongue clean; borels opened; pulse quicker than it was yesterday; slept but little. Did not take the beer. Continue the warm application, and take ten grains of Dover's powder at bed-time. The urine was much loaded with lithates.

17th. - Urine natural to-day; erysipelas has left the situa. tion of the groin, and the aneurism now occupies the middle of the thigh. Applied caustic around and over it in several places, and kept on the warm dressing. Take beer.

18th. - Slept well. The erysipelas does not extend, the caustic appears to have restrained its course. He looks anxious; pulse quick; tongue moist; appetite bad; took today and yesterday half a pint of beer. To have cod-liver oil and quinine, one tablespoonful three times a day.

19th. - Slept well. Erysipelas is better; appetite improved; pulse 100. Apply lint dipped in warm water, covered with oil-silk.

20th. - Improving. Takes nearly a quart of beer daily. Still much odema of the upper part of the thigh, but the erysipelas is nearly subsided.

21st.-Going on satisfactorily. Takes his food and beer well. Slight fluctuation on the inside of the thigh discovered to-day; but on passing a grooved needle no pus escaped.

27th. - A distinct point had formed, which fluctuated freely. He states that, for the last two days, he has suffered great pain, but did not call to his assistance any one during my absence from Cheltenham (which had been for three days), although I had ordered him to do so; he continued waiting for my return. The fluctuation was chiefly in the middle and inner part of the thigh. There was also a soft point in the situation of the sac: the pus was confined under the fascia. On making a deep incision at the depending point, a large quantity of pus, mixed with dark, grumous, coagulated por- 
tions of blood of a most offensive character, escaped; that portion situated in the sac of the aneurism communicated with, and was emptied by, the lower outlet. It was evident the sac had suppurated, and this was the original seat of the erysipelatous inflammation. I bandaged the upper portion of the thigh, using gentle pressure; and wet lint was kept on the depending point for the escape of the matter. His tongue was clean, and he had but little constitutional irritation. To take two grains of quinine three times a day, and as much Guinness's stout as he could comfortably drink, with generous diet.

28th.-Pulse quiet, but feeble. Scarcely any constitutional symptoms. About four ounces of pus discharged, of a more healthy character. I placed an india-rubber band between the two ends of the ligature, and strapped it up to the abdomen, my object being to keep steady and gentle extension on the ligature.

30th. - The discharge from the thigh is much diminished. Continues his porter and quinine, and is gradually gaining strength. He states that the ligature was pulled much by the band, and caused some pain during the night. On touching it I found it yield, and on replacing the band the ligature came away, this being the forty-sixth day from the operation, not including the day of its performance. That portion of the ligature which surrounded the artery readily allowed the passage of a good-sized goose-quill through it.

3Ist. - The discharge from the thigh is very trifling. Upon pressure around the spot from which the ligature escaped, a discharge of pus took place; pressure up the thigh also caused the same discharge from this point. He had no abdominal tenderness; bears pressure over the abdomen with ease.

April 12th. - Has gained strength; walks about; all discharge ceased both from the thigh and abdomen.

24th. - Quite well; the sac of the aneurism can barely be discovered. Upon very accurate examination, a small hard. ened spot is felt in its situation. The patient now walks sume distance daily, and says he feels strong; has gained much flesh; the foot and leg of their natural warmth; walks with the limb as strongly as ever; the circulation in it powerful, and perfectly re-established.

Cheltenham, 1856.

\section{ON THE}

\section{TREATMENT OF UTERINE DEVIATIONS.}

BY E. J. TILT, M.D.,

SEMTOR PHYSICIAN TO THE PARRINGDON GENERAL DISPINSAEX AND LYING-IN CEARITY.

(Continued from page 98.)

THE cases of uterine deviations which we are called upon to treat, are all complex problems-problems embracing various elements, each one of which has more particularly struck various practitioners, and guided their practice. The result has been, that one of the indications of such cases being often alone attended to, partial relief has alone been afforded. The radical cure will be more frequent when all the bearings of the case are taken into consideration. The only way of solving complicated problems is to eliminate one by one all their component elements; but before doing so I shall make a few remarks applicable to most of the cases under consideration. With regard to constitutional treatment I have nothing new to add, and its utility is obvious. The necessity of abstaining from connexion is not sufficiently borne in mind, and cannot in general be enforced. I have known patients who only suffered from uterine deformities for the day or two which followed connexion, and I have patients who are never well except when their husbands are absent for a few months. Thus there are some women so constituted that the reproductive organs cannot bear the matrimonial stimulus without the induction of disease. There is also another precept easy to explain and difficult to enforce-the considering the monthly period as one of disease. Some patients never suffer except during menstruation, a few days before and after. I have con siderably abated their sufferings, and sometimes removed them, by simply persuading them to remain in bed or on the sofa for a few days, using hip-baths and large warm abdominal poultices.
The importance I have attached to the relaxed state of the vagina in the mechanism of retroversion and anteversion, ex plain the importance I attach to such treatment as may correct this state. Astringent injections carefully made, either with Coxeter's syphon-syringe, or with Mr. Whitehead's prolapsus tube the changes being rung on strong solutions of alum sulphate of zinc, or tannin. The action of such remedies should be maintained by placing pledgets of cotton-wool, soaked in one of these solutions, high up in the vagina, removing these applications several times in the course of the day. There is another mode of applying astringents, which I consider even better than the above. I enclose in cotton-wool a little lump of alum or acetate of lead, about the size of a hazel-nut, and I tie round it a piece of string long enough for the ends to hang out of the pudenda, then the alum ball is placed as high as possible in the vagina, which may be done without using the speculum. The cotton-wool imbibes the vaginal fluids, and they dissolve the alum by degrees, which thus acts strongly for a long time on the walls of the vagina. So great is the astringent action, that it may become difficult to pass the finger through the vagina so as to reach the neck of the womb. I tell the patient to remove the cotton-wool on the second day, and to inject water freely, so as to bring away the thick coagulated mucus, which would diminish the effect of the sub. sequent injections. This mode of applying astringents $I$ have likewise found extremely useful in the treatment of many uterine affections, in that of metrorrhagia also, and I strongly advise others to try the plan. Cold water donches, both rectal and vaginal, act in the same way. If we examine carefully women who have suffered much from uterine deviations, they are found likewise complicated with inflammatcry congestions, erosions or ulcerations of the neck of the womb. The pathologist then infers that the pains experienced depend upon these inflammatory lesions. At all events, the indication is clear-to treat all ulcerations by the surgical modes of treatment on which I have dwelt in my work on "Diseases of Women," and to subdue inflammation, and by so doing the uterine deformity will; in some cases, be entirely removed, though in others the patient's sufferings will be only diminished. It is also equally true that in other patients the inflammatory affection of the neck of the womb may be cured, and yet they may still continue to suffer. The sequela of inflammation, the chronic enlargement of the neck of the womb or of its body, must not, however, be treated by halves, for there are patients who continue to suffer from nervous symptoms so long as there remains the smallest nucleus of organic mischief. A course of mercury or iodine may therefore be necessary, as well as local astringent measures.

On finding uterine deviations complicated by inflammatory lesions, I employ the measures necessary to cure them; and by so doing, I sometimes succeed in dispelling the symptoms. Having in like manner arrived at the conclusion that nterine deviations are of ten complicated by neuralgia, the indication is to treat such cases by the means found useful in neuralgic affections. Thus I have removed, or much alleviated, the sufferings of many affected with uterine deviations by the use of sedative injections into the bowels; generally prescribing from fifteen to thirty minims of Battley's solution, with a drachm of tincture of hyoscyamus, in a teacupful of warm milk.

No remedy is so effectual against the various forms of neuralgia as heat. The most efficacious remedy is the trans. current cauterization; but as the terrors which surround the red. hot iron render it very unacceptable in a sick room, we may seek to relieve by applying to the most painful spot a hammer previously plunged in boiling water. This was frecuently used by Sir Anthony Carlisle: it is known on the Continent as the "Marteau de Mlayor." Dr. Day has written on the use of this application in neuralgic and rheumatic affections; and Mr. Mitchell, of Dublin, has extensively used it for the neuralgic symptoms of uterine complaints. Jobert de Lamballe has published several cases of long-continued distressin symptoms, without any apparent structural change, and in which a cure was effected by passing the red-hot irou through the neck of the womb even into its cavity. Although unable to emulate the boldness of this practice, yet Paul Dubois intimates that the intra-uterine pessary has sometimes relieved patients by modifying the abnormal sensitiveness of the womb, just as the passage of a sound cures neuralgia of the neck of the bladder, or as the bistoury cures some neuralgic affections of the anus. In a case cited by Malgaigne, the woman was suddenly cured of all her sufferings by the application of the intra-uterine stem-pessary; but it was found that the deviation still remained the same.

The well-known constricting power of cold when perma. 